\title{
Linear Barycentric Rational Collocation Method for Beam Force Vibration Equation
}

\author{
Jin Li 1 and Yu Sang \\ College of Sciences, North China University of Science and Technology, Tangshan 063210, Hebei, China \\ Correspondence should be addressed to Jin Li; lijin@lsec.cc.ac.cn and Yu Sang; 1424833470@qq.com
}

Received 10 January 2021; Revised 29 January 2021; Accepted 16 February 2021; Published 25 February 2021

Academic Editor: Sandro Carbonari

Copyright (c) 2021 Jin Li and Yu Sang. This is an open access article distributed under the Creative Commons Attribution License, which permits unrestricted use, distribution, and reproduction in any medium, provided the original work is properly cited.

The linear barycentric rational collocation method for beam force vibration equation is considered. The discrete beam force vibration equation is changed into the matrix forms. With the help of convergence rate of barycentric rational interpolation, both the convergence rates of space and time can be obtained at the same time. At last, some numerical examples are given to validate our theorem.

\section{Introduction}

Beam vibration is the amount and direction of movement that a beam exhibits away from the point of applied force or the area of attachment. There are lots of application including the material used for the construction, length of the beam, construction of bridges, buildings, towers and the amount of force applied, and so on. Recently, applications of nanobeams in engineering structures $[1,2]$ like nonvolatile random access memory, nanotweezers, tunable oscillator, rotational motors, nanorelays, feedback-controlled nanocantilevers have also been developed.

There are lots of numerical methods [3-5] to solve the beam force vibration equation such as the finite difference method, finite element method, differential quadrature method, multiscale method, and spectral methods. The barycentric formula is studied in [6-8] and has been used to solve Volterra equation and Volterra integro-differential equation [9, 10]. Cirillo et al. [11-14] have proposed a rational interpolation scheme which has high numerical stability and interpolation accuracy on both equidistant and special distributed nodes. In [15-17], integro-differential equation, heat conduction equation, and biharnormic equation are solved by linear barycentric rational collocation method and the convergence rate is proved. In recent papers, Wang et al. [18-21] successfully applied the collocation method to solve initial value problems, plane elasticity problems, incompressible plane problems, and nonlinear problems which have expanded the application fields of the collocation method.

In this paper, we focus on the beam force vibration equation by barycentric rational interpolation methods. With the help of barycentric rational polynomial, the collocation scheme for beam force vibration equation and its matrix equation have been presented. The convergence rate of linear barycentric rational collocation methods has been proved. At last, two examples are presented to illustrate our theorem analysis.

\section{Collocation Scheme for Beam Force Vibration Equation}

In this article, we pay our attention to the numerical solution of beam force vibration as

$$
\rho A \frac{\partial^{2} u}{\partial t^{2}}+\mathrm{EI} \frac{\partial^{4} u}{\partial x^{4}}=q(x, t), \quad 0 \leq x \leq l ; t \geq 0 .
$$

By taking $a^{2}=(\mathrm{EI} / \rho A)$ and $f(x, t)=(q(x, t) / \rho A)$, we get the equation of EulerBernoulli beam as

$$
\frac{\partial^{2} u}{\partial t^{2}}+a^{2} \frac{\partial^{4} u}{\partial x^{4}}=f(x, t), \quad 0 \leq x \leq l ; t \geq 0,
$$


with boundary conditions as follows:

$$
\begin{aligned}
u(x, 0) & =\phi_{1}(x) ; \\
u_{t}(x, 0) & =\phi_{2}(x), \quad 0 \leq x \leq 1, \\
u(0, t) & =0, \\
u_{x x}(0, t) & =0, \quad t>0, \\
u(0, t) & =0, \\
u_{x}(0, t) & =0, \quad t>0, \\
u_{x x x}(0, t) & =0, \\
u_{x x}(0, t) & =0, \quad t>0 .
\end{aligned}
$$

The free vibration frequency of the beam is only related to the geometric and material parameters of the beam. The forced vibration of beam under external load is the result of superposition of free vibration and external excitation.

We partition the interval $[a, b]$ into $0=x_{0}<x_{1}<$ $\cdots<x_{m}=l, h=(l / m)$ and $[0, T]$ into $0=t_{0}<t_{1}<\cdots<t_{n}=$ $T, \tau=(T / n)$ with $\Omega=[0, l] \times[0, T]$ and. $\left(x_{i}, t_{j}\right), i=1,2$, $\ldots, m ; j=1,2, \ldots, n$.

We set

$$
u\left(x_{i}, t\right)=u_{i}(t), \quad i=0,1, \ldots, m,
$$

and its barycentric interpolation approximation is

$$
u(x, t)=\sum_{j=0}^{m} R_{j}(x) u_{j}(t)
$$

where

$$
R_{j}(x)=\frac{\left(w_{j} / x-x_{j}\right)}{\sum_{k=0}^{n}\left(w_{k} / x-x_{k}\right)}
$$

is the basis function, and

$$
w_{k}=\sum_{i \in J_{k}}(-1)^{i} \prod_{j=i, j \neq k}^{i+d} \frac{1}{x_{k}-x_{j}}
$$

is the weight function. Taking equation (5) into equation (2), we have

$$
\begin{aligned}
\sum_{j=0}^{m} R_{j}(x) \ddot{u}_{j}(t)+a^{2} \sum_{j=0}^{m} R_{j}^{(4)}(x) u_{j}(t) & =f(x, t), \\
\sum_{j=0}^{m} R_{j}\left(x_{i}\right) \ddot{u}_{j}(t)+a^{2} \sum_{j=0}^{m} R_{j}^{(4)}\left(x_{i}\right) u_{j}(t) & =f\left(x_{i}, t\right), \quad i=0,1, \ldots, m,
\end{aligned}
$$

then, we change the form into the following equation:

$$
\sum_{j=1}^{m} \delta_{i j} \ddot{u}_{j}(t)+a^{2} \sum_{j=1}^{m} C_{i j}^{(4)} u_{j}(t)=f_{i}(t), \quad i=0,1, \ldots, m,
$$

where $R_{j}\left(x_{i}\right)=\delta_{i j}, R_{j}^{\prime \prime}\left(x_{i}\right)=C_{i j}^{(2)}, C_{i j}^{(k)}, k=1,2 f\left(x_{i}, t\right)=f_{i}$ $(t), i=0,1, \ldots, m$. We get the matrix form as

$$
\left[\begin{array}{c}
\ddot{u}_{0}(t) \\
\vdots \\
\ddot{u}_{m}(t)
\end{array}\right]+a^{2}\left[\begin{array}{ccc}
C_{00}^{(4)} & \cdots & C_{0 m}^{(4)} \\
\vdots & & \vdots \\
C_{m 0}^{(4)} & \cdots & C_{m n}^{(4)}
\end{array}\right]\left[\begin{array}{c}
u_{0}(t) \\
\vdots \\
u_{m}(t)
\end{array}\right]=\left[\begin{array}{c}
f_{0}(t) \\
\vdots \\
f_{m}(t)
\end{array}\right],
$$

where $u(t)=\left[u_{0}(t), u_{1}(t), \ldots, u_{m}(t)\right]^{T}$ and $f(t)=\left[f_{0}(t)\right.$, $\left.f_{1}(t), \ldots, f_{m}(t)\right]^{T}$. By taking the notation,

$$
\begin{aligned}
& u_{i}\left(t_{j}\right)=u\left(x_{i}, t_{j}\right)=u_{i j}, \quad i=0,1, \ldots, m ; j=0,1, \ldots, n, \\
& u_{i}(t)=\sum_{k=0}^{n} R_{k}(t) u_{i k}, \quad i=0,1, \ldots, m
\end{aligned}
$$

we have

$$
\left[\begin{array}{c}
\sum_{k=0}^{n} \ddot{R}_{k}\left(t_{j}\right) u_{0 k} \\
\vdots \\
\sum_{k=0}^{n} \ddot{R}_{k}\left(t_{j}\right) u_{m k}
\end{array}\right]+a^{2}\left[\begin{array}{ccc}
C_{00}^{(4)} & \cdots & C_{\mid m}^{(4)} \\
\vdots & & \vdots \\
C_{m 0}^{(4)} & \cdots & C_{m n}^{(4)}
\end{array}\right]\left[\sum_{k=0}^{n} R_{k}\left(t_{j}\right) u_{0 k} \vdots \sum_{k=0}^{n} R_{k}\left(t_{j}\right) u_{n k}\right]=\left[\begin{array}{c}
f_{0}\left(t_{j}\right) \\
\vdots \\
f_{m}\left(t_{j}\right)
\end{array}\right]
$$

where $j=0,1, \ldots, n$.

Its matrix form can be expressed as

$$
\left[\left(I_{n} \otimes D^{(2)}\right)+a^{2}\left(C^{(4)} \otimes I_{n}\right)\right] U=F .
$$

The matrix equation can also be written as

$$
\mathrm{LU}=F,
$$

where $L=\left(I_{n} \otimes D^{(2)}\right)+a^{2}\left(C^{(4)} \otimes I_{n}\right)$ and $\otimes$ is Kronecher product of matrix:

$$
\begin{aligned}
U & =\left[u_{00}, u_{01}, \ldots, u_{0 n}, u_{10}, u_{11}, \ldots, u_{1 n}, \ldots, u_{m 0}, u_{m 1}, \ldots, u_{m n}\right]^{T}, \\
F & =\left[f_{00}, f_{01}, \ldots, f_{0 n}, f_{10}, f_{11}, \ldots, f_{1 n}, \ldots, f_{m 0}, f_{m 1}, \ldots, f_{m n}\right]^{T},
\end{aligned}
$$

$f_{i j}=f\left(x_{i}, t_{j}\right), i=0,1, \ldots, m ; j=0,1, \ldots, n$, and 


$$
\begin{aligned}
& C_{i j}^{(4)}=r_{i}^{(4)}\left(x_{j}\right), \\
& C_{i j}^{(2)}=r_{i}^{\prime \prime}\left(x_{j}\right), \\
& D_{i j}^{(2)}=r_{i}^{\prime \prime}\left(t_{j}\right),
\end{aligned}
$$

are the elements of the differentiation matrices with

$$
\begin{aligned}
& r_{i}^{\prime}\left(t_{j}\right)=\frac{w_{i} / w_{j}}{t_{j}-t_{i}}, \quad i \neq j, \\
& r_{i}^{\prime}\left(t_{i}\right)=-\sum_{j \neq i} r_{i}^{\prime}\left(t_{j}\right), \quad i=j .
\end{aligned}
$$

Similarly, we have

$$
\begin{aligned}
& r_{j}^{\prime}\left(x_{i}\right)=\frac{w_{j} / w_{i}}{x_{i}-x_{k}}, \quad j \neq i, \\
& r_{j}^{\prime}\left(x_{i}\right)=-\sum_{j \neq i} r_{j}^{\prime}\left(x_{i}\right), \quad i=j, \\
& r_{j}^{\prime \prime}\left(x_{i}\right)=-2 \frac{w_{j} / w_{i}}{x_{i}-x_{j}}\left(\sum_{k \neq i} \frac{w_{k} / w_{i}}{x_{i}-x_{k}}+\frac{1}{x_{i}-x_{j}}\right), \quad j \neq i, \\
& r_{i}^{\prime \prime}\left(x_{i}\right)=-\sum_{j \neq i} r_{j}^{\prime \prime}\left(x_{i}\right), \quad i=j,
\end{aligned}
$$

for $k \geq 2$, according to mathematical induction, we obtain the recurrence formula of $m$-order differential matrix as

$$
\begin{aligned}
& \left\{\begin{aligned}
C_{i j}^{(4)}=4\left(C_{i i}^{(3)} C_{i j}^{(1)}-\frac{C_{i j}^{(3)}}{x_{i}-x_{j}}\right), & i \neq j \\
& C_{i j}^{(4)},
\end{aligned}\right. \\
& C_{i i}^{(4)}=-\sum_{j=1, j \neq i}^{n} \\
& \left\{\begin{array}{cc}
D_{i j}^{(2)}=2\left(D_{i i}^{(1)} D_{i j}^{(1)}-\frac{D_{i j}^{(1)}}{t_{i}-t_{j}}\right), & i \neq j, \\
D_{i i}^{(2)}=-\sum_{j=1, j \neq i}^{n} & D_{i j}^{(2)} .
\end{array}\right.
\end{aligned}
$$

\section{Convergence and Error Analysis}

For the barycentric rational interpolants of function $f(x)$ with $r(x)$, its error convergence rate is

$$
\begin{aligned}
& e(x):=f(x)-r(x)=\left(x-x_{i}\right), \ldots,\left(x-x_{i+d}\right) f\left[x_{i}, x_{i+1}, \ldots, x_{i+d}, x\right], \\
& e(x):=\max _{a \leq x \leq b} e(x)=\frac{\sum_{i=0}^{n-d} \lambda_{i}(x)\left(f(x)-p_{i}(x)\right)}{\sum_{i=0}^{n-d} \lambda_{i}(x)}=\frac{A(x)}{B(x)}=O\left(h^{d+1}\right),
\end{aligned}
$$

where $d$ is the degree of polynomial $r(x)$ :

$$
\begin{aligned}
& A(x):=\sum_{i=0}^{n-d}(-1)^{i} f\left[x_{i}, \ldots, x_{i+d}, x\right], \\
& B(x):=\sum_{i=0}^{n-d} \lambda_{i}(x),
\end{aligned}
$$

where

$$
\lambda_{i}(x)=\frac{(-1)^{i}}{\left(x-x_{i}\right), \ldots,\left(x-x_{i+d}\right)} .
$$

For the barycentric rational interpolants of function $u(x, t)$ with $r_{m, n}(x, t)$, we can get the barycentric rational interpolants:

$$
r_{m, n}(x, t)=\frac{\sum_{i=0}^{m} \sum_{j=0}^{n}\left(w_{i, j} /\left(x-x_{i}\right)\left(t-t_{j}\right)\right) u_{i, j}}{\sum_{i=0}^{m} \sum_{j=0}^{n}\left(w_{i, j} /\left(x-x_{i}\right)\left(t-t_{j}\right)\right)}
$$

where

$$
w_{i, j}=(-1)^{i-d_{1}+j-d_{2}} \sum_{k_{1} \in J_{i}} \prod_{h_{1}=k_{1}, h_{1} \neq j}^{k_{1}+d_{1}} \frac{1}{\left|x_{i}-x_{h_{1}}\right|} \sum_{k_{2} \in J_{j}} \prod_{h_{2}=k_{2}, h_{2} \neq j}^{k_{2}+d_{2}} \frac{1}{\left|t_{j}-t_{h_{2}}\right|},
$$


$J_{i}=\left\{k_{1} \in I_{m}: i-d_{1} \leq k_{1} \leq i\right\}, I_{m}=\left\{0, \ldots, m-d_{1}\right\}, J_{j}=\left\{k_{2}\right.$

$\left.\in I_{n}: j-d_{2} \leq k_{2} \leq j\right\}, I_{n}=\left\{0, \ldots, n-d_{2}\right\}$, and $d_{1}$ and $d_{2}$ are the degree of polynomial of $r_{m, n}(x, t)$.

$$
\begin{aligned}
& e(x, t):=u(x, t)-r_{m, n}(x, t)=\left(x-x_{i}\right), \ldots,\left(x-x_{i+d_{1}}\right) u\left[x_{i}, x_{i+1}, \ldots, x_{i+d_{1}}, x\right] \\
& +\left(t-t_{j}\right), \ldots,\left(t-t_{j+d_{2}}\right) u\left[t_{j}, t_{j+1}, \ldots, t_{j+d_{2}}, t\right] \text {. } \\
& |e(x, t)| \leq C\left(h^{d_{1}+1}+\tau^{d_{2}+1}\right) .
\end{aligned}
$$

The following lemma has been proved by Jean-Paul Berrut in [10].

Lemma 1 (see reference [10]). For the $e(x)$ defined in equation (20), we have

Proof. For $(x, t)$, the function $w_{i, j}(x, t)$ is well-defined, and the error functional can be expressed as

$$
\begin{cases}|e(x)| \leq C h^{d+1}, & u \in C^{d+2}[a, b], \\ \left|e^{\prime}(x)\right| \leq C h^{d}, & u \in C^{d+3}[a, b], \\ \left|e^{\prime \prime}(x)\right| \leq C h^{d-1}, & u \in C^{d+4}[a, b], d \geq 1 .\end{cases}
$$

$$
u(x, t)-r_{m, n}(x, t)=\frac{\sum_{i=0}^{n_{1}-d_{1}} \sum_{j=0}^{n_{2}-d_{2}} \lambda_{i}(x) \lambda_{j}(t)\left(u(x, t)-r_{m, n}(x, t)\right)}{\sum_{i=0}^{n_{1}-d_{1}} \sum_{j=0}^{n_{2}-d_{2}} \lambda_{i}(x) \lambda_{j}(t)},
$$

By the Newton error formula,

Theorem 1. For the $e(x, t)$ defined in equation (26) and $u(x, t) \in C^{d_{1}+2}[a, b] \times C^{d_{2}+2}[0, T]$, we have

$$
\begin{aligned}
u(x, t)-r_{m, n}(x, t)= & u(x, t)-u_{1}(x, t)+u_{1}(x, t)-r_{m, n}(x, t) \\
= & \left(x-x_{i}\right), \ldots,\left(x-x_{i+d_{1}}\right) u\left[x_{i}, x_{i+1}, \ldots, x_{i+d_{1}}, x, t\right] \\
& +\left(t-t_{j}\right), \ldots,\left(t-t_{j+d_{2}}\right) u\left[t_{j}, t_{j+1}, \ldots, t_{j+d_{2}}, x, t\right], \\
& \left|\sum_{i=0}^{n_{1}-d_{1}} \lambda_{i}(x)\right| \geq \frac{1}{d_{1} ! h^{d_{1}+1}}, \\
\sum_{i=0}^{n_{1}-d_{1}} \lambda_{i}(x) & \left|\sum_{j=0}^{n_{2}-d_{2}} \lambda_{j}(t)\right| \geq \frac{1}{d_{2} ! \tau^{d_{2}+1}} .
\end{aligned}
$$

we reach that

$$
\begin{aligned}
u(x, t)-r_{m, n}(x, t)= & \frac{\sum_{i=0}^{n_{1}-d_{1}}(-1)^{i} u\left[x_{i}, x_{i+1}, \ldots, x_{i+d_{1}}, x, t\right]}{\sum_{i=0}^{n_{1}-d_{1}} \lambda_{i}(x)} \\
& +\frac{\sum_{j=0}^{n_{2}-d_{2}}(-1)^{j} u\left[t_{j}, t_{j+1}, \ldots, t_{j+d_{2}}, x, t\right]}{\sum_{j=0}^{n_{2}-d_{2}} \lambda_{j}(t)} .
\end{aligned}
$$

By the similarly analysis in Li and Cheng [15], we have

Combining equations (29)-(31) together, the proof of Theorem 1 is completed.

Corollary 1. For the e $(x, t)$ defined in equation (26), we have

$$
\begin{cases}\left|e_{t t}(x, t)\right| \leq C\left(h^{d_{1}+1}+\tau^{d_{2}-1}\right), & u(x, t) \in C^{d_{1}+3}[a, b] \times C^{d_{2}+2}[0, T], d_{2} \geq 1 \\ \left|e_{x x x x}(x, t)\right| \leq C\left(h^{d_{1}-3}+\tau^{d_{2}+1}\right), & u(x, t) \in C^{d_{1}+4}[a, b] \times C^{d_{2}+2}[0, T], d_{1} \geq 3\end{cases}
$$

Taking the numerical scheme,

$$
\sum_{j=1}^{n} u_{j} R_{j}^{\prime \prime}(x, t)+a^{2} \sum_{j=1}^{n} u_{j} R_{j}^{(4)}(x, t)=f(x, t)
$$

\section{Combining equations (1) and (34), we have}

$$
T e(x):=e^{\prime \prime}(x, t)+a^{2} e^{(4)}(x, t)=R_{f}(x, t),
$$

where $R_{f}(x, t)=f(x, t)-R_{f}\left(x_{k}, t\right), k=1,2, \cdots, n$. 
Based on the above lemma, we get the following where theorem.

$$
\begin{aligned}
& R_{1}(x, t)=u_{t t}(x, t)-u_{t t}\left(x_{m}, t_{n}\right), \\
& R_{2}(x, t)=a^{2}\left[u_{x x x x}(x, t)-u_{x x x x}\left(x_{m}, t_{n}\right)\right] .
\end{aligned}
$$

Theorem 2. Let $R_{f}(x, t)=f(x, t)-R_{f}\left(x_{k}, t\right), k=1,2, \ldots$, $n$, we have

$$
\left|u(x, t)-u\left(x_{m}, t_{n}\right)\right| \leq C\left(h^{d_{1}-3}+\tau^{d_{2}-1}\right) .
$$

As for the $R_{1}(x, t)$, we have

Proof. As

$$
\begin{aligned}
& u_{t t}(x, t)+a^{2} u_{x x x x}(x, t)-\left[u_{t t}\left(x_{m}, t_{n}\right)+a^{2} u_{x x x x}\left(x_{m}, t_{n}\right)\right] \\
& \quad=u_{t t}(x, t)-u_{t t}\left(x_{m}, t_{n}\right)+a^{2}\left[u_{x x x x}(x, t)-u_{x x x x}\left(x_{m}, t_{n}\right)\right] \\
& \quad:=R_{1}(x, t)+R_{2}(x, t)
\end{aligned}
$$

$$
\begin{aligned}
R_{1}(x, t) & =u_{t t}(x, t)-u_{t t}\left(x_{m}, t_{n}\right) \\
& =u_{t t}(x, t)-u_{t t}\left(x_{m}, t\right)+u_{t t}\left(x_{m}, t\right)-u_{t t}\left(x_{m}, t_{n}\right) \\
& =s \frac{\sum_{i=0}^{m-d_{1}}(-1)^{i} u_{t t}\left[x_{i}, x_{i+1}, \ldots, x_{i+d_{1}}, x, t\right]}{\sum_{i=0}^{m-d_{1}} \lambda_{i}(x)}+\frac{\sum_{j=0}^{n-d_{2}}(-1)^{j} u_{t t}\left[t_{j}, t_{j+1}, \ldots, t_{j+d_{2}}, x, t\right]}{\sum_{j=0}^{n-d_{2}} \lambda_{j}(t)} \\
& =e_{t t}\left(x, t_{n}\right)+e_{t t}\left(x_{m}, t_{n}\right) .
\end{aligned}
$$

Similarly, for $R_{2}(x, t)$, we have

$$
R_{2}(x, t)=u_{x x x x}(x, t)-u_{x x x x}\left(x_{m}, t_{n}\right)=e_{x x x x}\left(x, t_{n}\right)-e_{x x x x}\left(x_{m}, t_{n}\right) .
$$

Then, we have

$$
u(x, 0)=0, \quad 0<x<1
$$

$\left|u(x, t)-u\left(x_{m}, t_{n}\right)\right| \leq\left|\sum_{j=1} M_{j}(x)\right||T e(x)| \leq C\left(h^{d_{1}-3}+\tau^{d_{2}-1}\right)$.

The proof is completed.

\section{Numerical Examples}

Example 1. Consider the beam force vibration equation:

$$
\frac{\partial^{2} u}{\partial t^{2}}+\left(\frac{1}{x}+\frac{x^{4}}{120}\right) \frac{\partial^{4} u}{\partial x^{4}}=0, \quad \frac{1}{2} \leq x \leq 1 ; t \geq 0,
$$

with the following conditions: $u_{t}(x, 0)=1+\frac{x^{5}}{120}, \quad \frac{1}{2} \leq x \leq 1$,

$u\left(\frac{1}{2}, t\right)=\left(1+\frac{0.5^{5}}{120}\right) \sin t$

$u_{x x}\left(\frac{1}{2}, t\right)=\frac{1}{48} \sin t$

$u(1, t)=\frac{121}{120} \sin t$

$u_{x x}(1, t)=\frac{1}{6} \sin t$

Its analysis solutions is 
TABLE 1: Convergence rate of equidistant nodes with different $d_{1}\left(d_{2}=7\right)$ values.

\begin{tabular}{|c|c|c|c|c|c|c|c|c|}
\hline$n$ & \multicolumn{2}{|c|}{$d_{1}=2$} & \multicolumn{2}{|c|}{$d_{1}=3$} & \multicolumn{2}{|c|}{$d_{1}=4$} & \multicolumn{2}{|c|}{$d_{1}=5$} \\
\hline $8 \times 8$ & $\begin{array}{c}3.5062 e-0 \\
4\end{array}$ & & $1.7148 e-04$ & & $2.7309 e-05$ & & $2.7309 e-05$ & \\
\hline $16 \times 16$ & $\begin{array}{c}2.1219 e-0 \\
4\end{array}$ & $\begin{array}{c}0.7532 \\
6\end{array}$ & $4.1154 e-05$ & $\begin{array}{c}2.087 \\
7\end{array}$ & $3.6634 e-08$ & $\begin{array}{c}9.570 \\
7\end{array}$ & $3.6630 e-08$ & $\begin{array}{c}9.570 \\
8\end{array}$ \\
\hline $32 \times 32$ & $\begin{array}{c}1.1641 e-0 \\
4\end{array}$ & $\begin{array}{c}0.8721 \\
9\end{array}$ & $9.9209 e-06$ & $\begin{array}{c}2.058 \\
6\end{array}$ & $6.9359 e-11$ & $\begin{array}{c}9.050 \\
9\end{array}$ & $9.5628 e-11$ & 8.5875 \\
\hline $64 \times 64$ & $\begin{array}{c}6.0977 e-0 \\
5\end{array}$ & $\begin{array}{c}0.9343 \\
2\end{array}$ & $2.4447 e-06$ & $\begin{array}{c}2.022 \\
2\end{array}$ & $5.7157 e-07$ & - & $3.7940 e-07$ & - \\
\hline
\end{tabular}

TABLE 2: Convergence rate of equidistant nodes with different $d_{2}\left(d_{1}=7\right)$ values.

\begin{tabular}{|c|c|c|c|c|c|c|c|c|}
\hline$n$ & \multicolumn{2}{|c|}{$d_{1}=2$} & \multicolumn{2}{|c|}{$d_{1}=3$} & \multicolumn{2}{|c|}{$d_{1}=4$} & \multicolumn{2}{|c|}{$d_{1}=5$} \\
\hline $8 \times 8$ & $\begin{array}{c}7.5819 e-0 \\
3\end{array}$ & & $1.3995 e-03$ & & $4.6760 e-04$ & & $1.7882 e-04$ & \\
\hline $16 \times 16$ & $\begin{array}{c}7.9829 e-0 \\
4\end{array}$ & 3.2763 & $4.7529 e-05$ & $\begin{array}{c}4.908 \\
7\end{array}$ & $1.3026 e-05$ & $\begin{array}{c}5.194 \\
5\end{array}$ & $1.1934 e-06$ & 7.2560 \\
\hline $32 \times 32$ & $\begin{array}{c}9.0685 e-0 \\
5\end{array}$ & 3.1440 & $2.0817 e-06$ & $\begin{array}{c}4.519 \\
1\end{array}$ & $4.3378 e-07$ & $\begin{array}{c}4.914 \\
4\end{array}$ & $1.3851 e-07$ & 3.1131 \\
\hline $64 \times 64$ & $\begin{array}{c}1.6500 e-0 \\
5\end{array}$ & 2.4598 & $1.5216 e-06$ & - & $1.0236 e-06$ & - & $6.4063 e-07$ & - \\
\hline
\end{tabular}

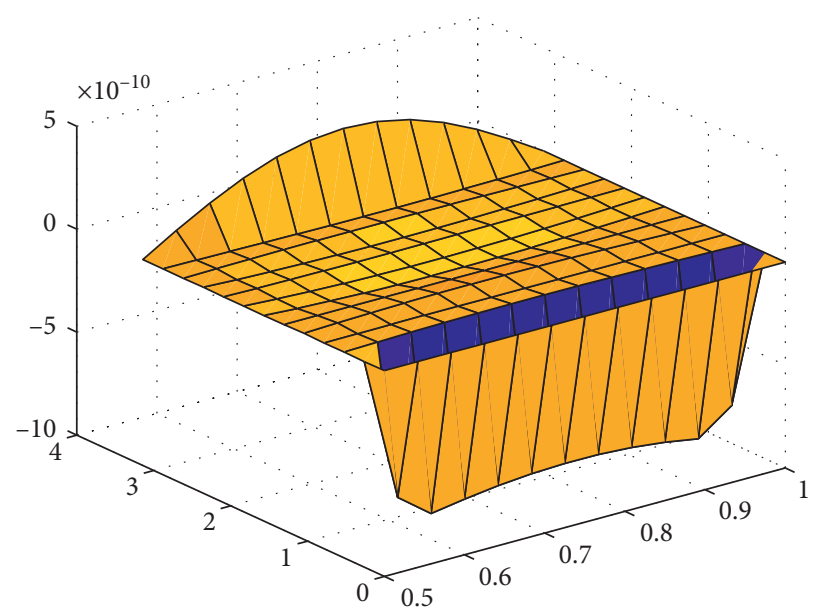

FIGURE 1: Errors of LBRCM by equidistant nodes with $m=n=12$ and $d_{1}=d_{2}=9$.

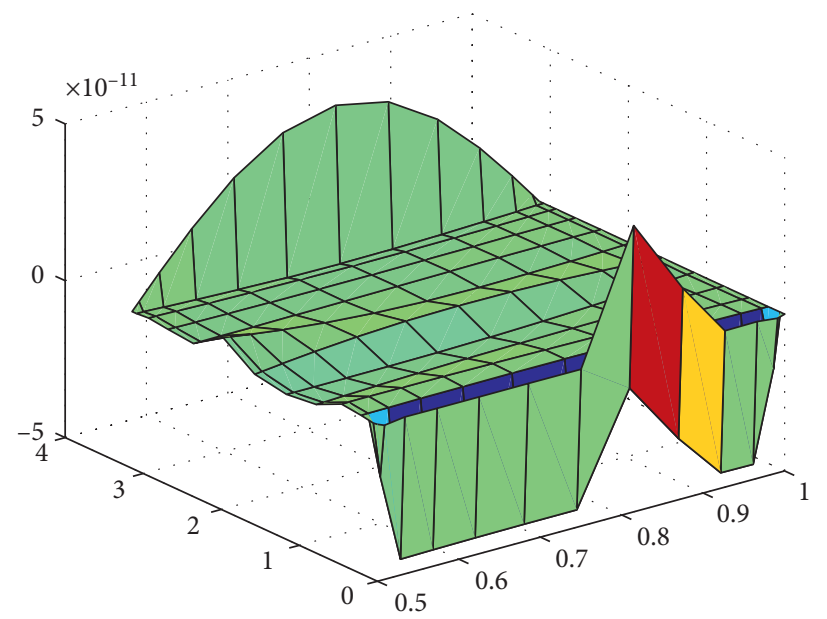

FIGURE 2: Errors of LBRCM by quasi-equidistant nodes with $m=n=12$ and $d_{1}=d_{2}=9$. 


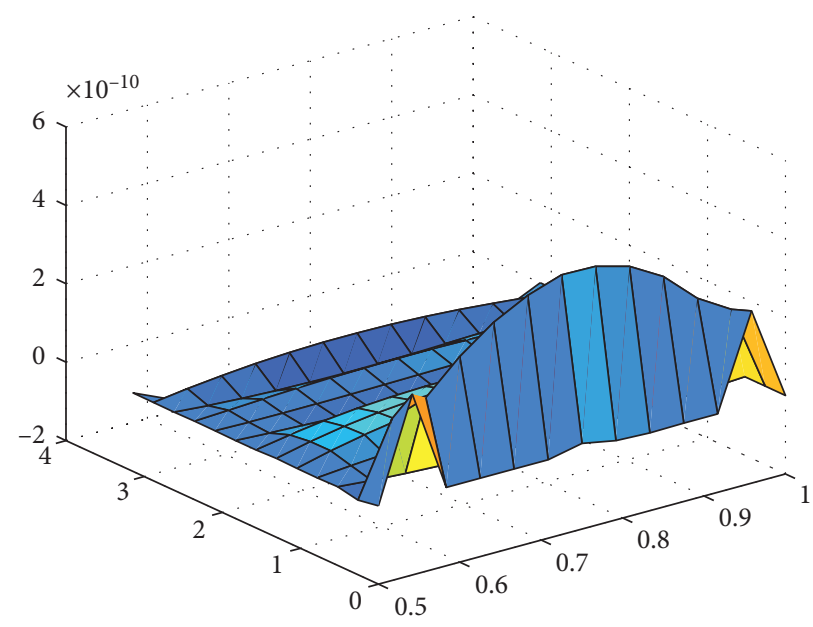

FIGURE 3: Errors of equidistant nodes with $m=n=12$.

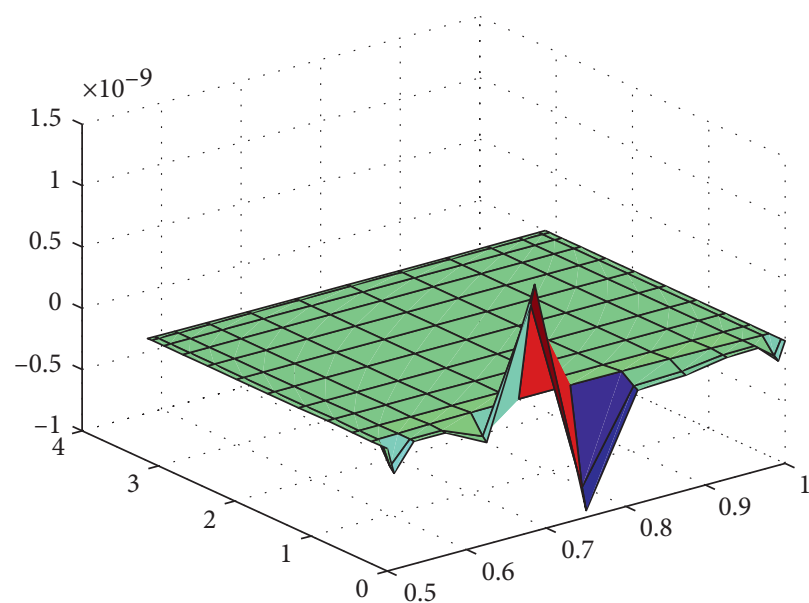

FIGURE 4: Errors of quasi-equidistant nodes with $m=n=12$.

TAвLE 3: Convergence rate of quasi-equidistant nodes with different $d_{1}\left(d_{2}=7\right)$ values.

\begin{tabular}{|c|c|c|c|c|c|c|c|c|}
\hline$n$ & $d_{1}=2$ & & $d_{1}=3$ & & $d_{1}=4$ & & $d_{1}=5$ & \\
\hline $8 \times 8$ & $\begin{array}{c}1.4433 e-0 \\
4\end{array}$ & & $1.7229 e-04$ & & $9.1628 e-06$ & & $9.1628 e-06$ & \\
\hline $16 \times 16$ & $\begin{array}{c}1.6054 e-0 \\
4\end{array}$ & - & $7.4842 e-07$ & $\begin{array}{c}7.917 \\
2\end{array}$ & $8.0479 e-10$ & $\begin{array}{c}13.54 \\
5\end{array}$ & $1.6691 e-09$ & 12.493 \\
\hline $32 \times 32$ & $\begin{array}{c}7.6781 e-0 \\
5\end{array}$ & 1.0791 & $6.9197 e-06$ & - & $1.4486 e-06$ & - & $1.5017 e-05$ & - \\
\hline $64 \times 64$ & $\begin{array}{c}1.4474 e-0 \\
2\end{array}$ & - & $\begin{array}{c}3.4807 e+0 \\
0\end{array}$ & - & $8.0897 e-01$ & - & $1.1143 e-01$ & - \\
\hline
\end{tabular}

TABLE 4: Convergence rate of quasi-equidistant nodes with different $d_{2}\left(d_{1}=7\right)$ values.

\begin{tabular}{|c|c|c|c|c|c|c|c|c|}
\hline$n$ & $d_{1}=$ & & $d_{1}=3$ & & $d_{1}=$ & & $d_{1}=5$ & \\
\hline $8 \times 8$ & $\begin{array}{c}1.2079 e-0 \\
3\end{array}$ & & $1.9645 e-04$ & & $1.1008 e-04$ & & $4.7330 e-05$ & \\
\hline $16 \times 16$ & $\begin{array}{c}2.6034 e-0 \\
5\end{array}$ & 5.6064 & $6.9740 e-07$ & $\begin{array}{c}8.208 \\
4\end{array}$ & $2.0174 e-07$ & $\begin{array}{c}9.162 \\
2\end{array}$ & $1.0751 e-08$ & 12.1751 \\
\hline $32 \times 32$ & $\begin{array}{c}1.2548 e-0 \\
3\end{array}$ & - & $8.6849 e-07$ & - & $7.2689 e-06$ & - & $5.8886 e-06$ & - \\
\hline $64 \times 64$ & $5.1823 e-0$ & 4.6011 & $1.0338 e-03$ & - & $7.2953 e-03$ & - & $5.1897 e-03$ & - \\
\hline
\end{tabular}




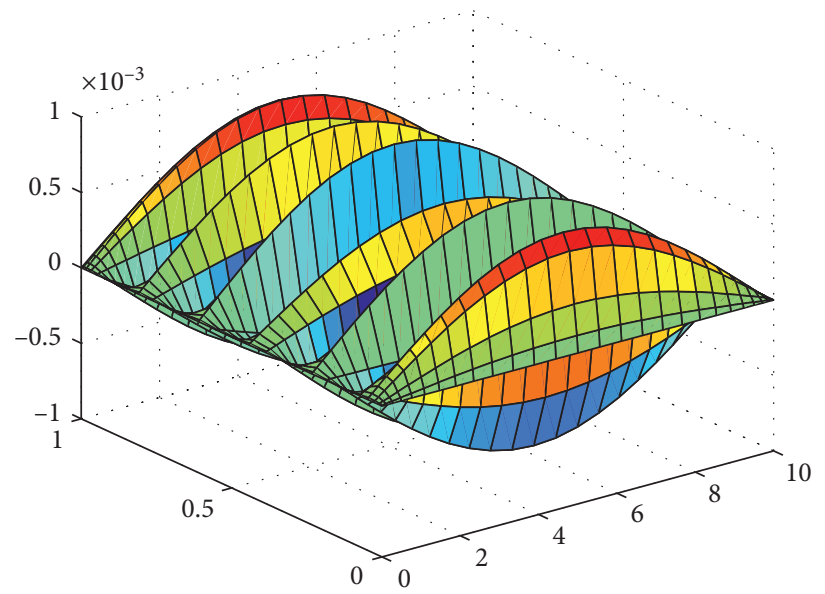

FIgURE 5: Errors of deflection with quasi-equidistant nodes $m=n=28$.

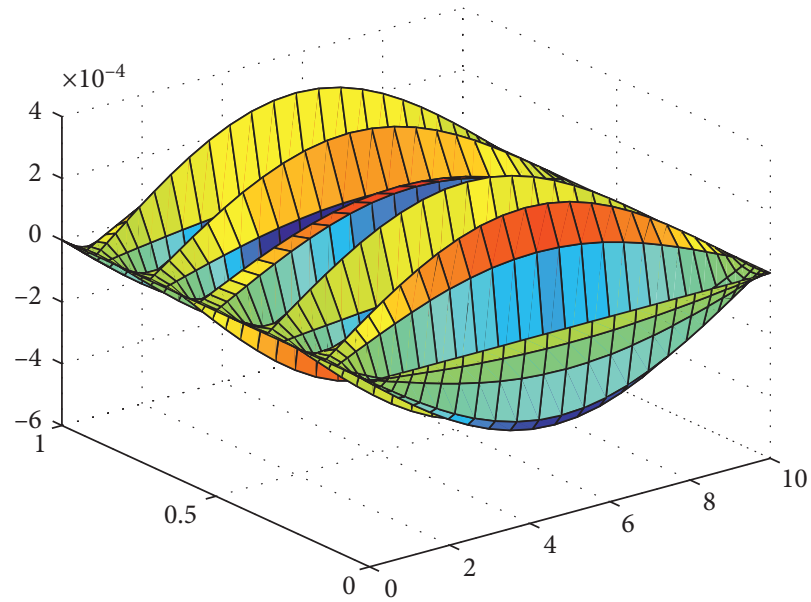

Figure 6: Errors of deflection with quasi-equidistant nodes $m=n=28$ and $d_{1}=d_{2}=25$.

TABLE 5: Convergence rate of deflection with equidistant nodes $d_{1}\left(d_{2}=7\right)$.

\begin{tabular}{|c|c|c|c|c|c|c|c|c|}
\hline$n$ & \multicolumn{2}{|c|}{$d_{1}=2$} & \multicolumn{2}{|c|}{$d_{1}=3$} & \multicolumn{2}{|c|}{$d_{1}=4$} & \multicolumn{2}{|c|}{$d_{1}=5$} \\
\hline $8 \times 8$ & $\begin{array}{c}2.3676 e+0 \\
2\end{array}$ & & $\begin{array}{c}3.7721 e+0 \\
2\end{array}$ & & $\begin{array}{c}4.9328 e+0 \\
2\end{array}$ & & $4.7610 e+02$ & \\
\hline $16 \times 16$ & $\begin{array}{c}4.1793 e+0 \\
1\end{array}$ & 2.5021 & $\begin{array}{c}1.3027 e+0 \\
3\end{array}$ & - & $\begin{array}{c}6.6585 e+0 \\
2\end{array}$ & - & $7.5280 e+02$ & - \\
\hline $32 \times 32$ & $\begin{array}{c}5.2574 e+0 \\
1\end{array}$ & - & $\begin{array}{c}3.3896 e+0 \\
0\end{array}$ & $\begin{array}{c}8.586 \\
2\end{array}$ & $\begin{array}{c}1.5076 e+0 \\
0\end{array}$ & $\begin{array}{c}8.786 \\
8\end{array}$ & $1.2155 e+00$ & 9.2746 \\
\hline $64 \times 64$ & $\begin{array}{c}3.7355 e+0 \\
1\end{array}$ & - & $7.0233 e-01$ & $\begin{array}{c}2.270 \\
9\end{array}$ & $1.0927 e-01$ & $\begin{array}{c}3.786 \\
3\end{array}$ & $4.0207 e-02$ & 4.9179 \\
\hline
\end{tabular}

TABLE 6: Convergence rate of bending moment with equidistant nodes $d_{1}\left(d_{2}=7\right)$.

\begin{tabular}{|c|c|c|c|c|c|c|c|c|}
\hline$n$ & $d_{1}=1$ & & $d_{1}=3$ & & $d_{1}=4$ & & $d_{1}=5$ & \\
\hline $8 \times 8$ & 8.6319 & & $\begin{array}{c}1.2547 e+0 \\
1\end{array}$ & & $\begin{array}{c}1.5671 e+0 \\
1\end{array}$ & & $1.5214 e+01$ & \\
\hline $16 \times 16$ & $6.7548 e-01$ & 3.6757 & $\begin{array}{c}2.1839 e+0 \\
1\end{array}$ & - & $\begin{array}{c}1.1063 e+0 \\
1\end{array}$ & $\begin{array}{c}0.502 \\
4\end{array}$ & $1.2536 e+01$ & - \\
\hline $32 \times 32$ & $8.5357 e-01$ & - & $5.4495 e-02$ & $\begin{array}{c}8.646 \\
6\end{array}$ & $2.4465 e-02$ & $\begin{array}{c}8.820 \\
8\end{array}$ & $1.9834 e-02$ & 9.3039 \\
\hline $64 \times 64$ & $6.0317 e-01$ & 0.5009 & $1.1256 e-02$ & $\begin{array}{c}2.275 \\
5\end{array}$ & $1.7640 e-03$ & $\begin{array}{c}3.793 \\
8\end{array}$ & $6.5673 e-04$ & 4.9165 \\
\hline
\end{tabular}


TABLE 7: Convergence rate of deflection with equidistant nodes $d_{2}\left(d_{1}=7\right)$.

\begin{tabular}{|c|c|c|c|c|c|c|c|c|}
\hline \multirow{2}{*}{$\frac{n}{8 \times 8}$} & \multicolumn{2}{|c|}{$d_{1}=2$} & \multicolumn{2}{|c|}{$d_{1}=3$} & \multicolumn{2}{|c|}{$d_{1}=4$} & \multicolumn{2}{|c|}{$d_{1}=5$} \\
\hline & $\begin{array}{c}1.2258 e+0 \\
2\end{array}$ & & $\begin{array}{c}1.7906 e+0 \\
2\end{array}$ & & $\begin{array}{c}2.5725 e+0 \\
2\end{array}$ & & $3.5441 e+02$ & \\
\hline $16 \times 16$ & $\begin{array}{c}2.5305 e+0 \\
1\end{array}$ & 2.2762 & $\begin{array}{c}2.2997 e+0 \\
1\end{array}$ & $\begin{array}{c}2.960 \\
9\end{array}$ & $\begin{array}{c}6.5860 e+0 \\
1\end{array}$ & $\begin{array}{c}1.965 \\
7\end{array}$ & $5.9839 e+01$ & 2.5662 \\
\hline $32 \times 32$ & $\begin{array}{c}3.2139 e+0 \\
0\end{array}$ & 2.9770 & $\begin{array}{c}4.7977 e+0 \\
0\end{array}$ & $\begin{array}{c}2.261 \\
0\end{array}$ & $\begin{array}{c}1.2807 e+0 \\
0\end{array}$ & $\begin{array}{c}5.684 \\
4\end{array}$ & $6.7902 e+00$ & 3.1396 \\
\hline $64 \times 64$ & $9.0199 e-01$ & 1.8331 & $3.2947 e-01$ & $\begin{array}{c}3.864 \\
1\end{array}$ & $2.3183 e-01$ & $\begin{array}{c}2.465 \\
8\end{array}$ & $1.4751 e-01$ & 5.5245 \\
\hline
\end{tabular}

TABLE 8: Convergence rate of bending moment with equidistant nodes $d_{2}\left(d_{1}=7\right)$.

\begin{tabular}{|c|c|c|c|c|c|c|c|c|}
\hline$n$ & \multicolumn{2}{|c|}{$d_{1}=2$} & \multicolumn{2}{|c|}{$d_{1}=3$} & \multicolumn{2}{|c|}{$d_{1}=4$} & \multicolumn{2}{|c|}{$d_{1}=5$} \\
\hline $8 \times 8$ & $\begin{array}{c}3.9561 e+0 \\
0\end{array}$ & & $\begin{array}{c}05.7800 e+0 \\
0\end{array}$ & & $\begin{array}{c}8.3046 e+0 \\
0\end{array}$ & & $1.1442 e+01$ & \\
\hline $16 \times 16$ & $4.2155 e-01$ & 3.2303 & $3.8310 e-01$ & $\begin{array}{c}3.915 \\
3\end{array}$ & $\begin{array}{c}1.0971 e+0 \\
0\end{array}$ & $\begin{array}{c}2.920 \\
2\end{array}$ & $9.9683 e-01$ & 3.5208 \\
\hline $32 \times 32$ & $5.2460 e-02$ & 3.0064 & $7.8314 e-02$ & $\begin{array}{c}2.290 \\
4\end{array}$ & $2.0905 e-02$ & $\begin{array}{c}5.713 \\
7\end{array}$ & $1.1084 e-01$ & 3.1689 \\
\hline $64 \times 64$ & $1.4697 e-02$ & 1.8357 & $5.3686 e-03$ & $\begin{array}{c}3.866 \\
6\end{array}$ & $3.7775 e-03$ & $\begin{array}{c}2.468 \\
3\end{array}$ & $2.4037 e-03$ & 5.5271 \\
\hline
\end{tabular}

TABLE 9: Convergence rate of deflection with quasi-equidistant nodes $d_{1}\left(d_{2}=7\right)$.

\begin{tabular}{|c|c|c|c|c|c|c|c|c|}
\hline$n$ & $d_{1}=2$ & & $d_{1}=$ & & $d_{1}=$ & & $d_{1}=$ & \\
\hline $8 \times 8$ & $\begin{array}{c}4.1355 e+0 \\
2\end{array}$ & & $\begin{array}{c}4.0577 e+0 \\
2\end{array}$ & & $\begin{array}{c}4.3916 e+0 \\
2\end{array}$ & & $4.3806 e+02$ & \\
\hline $16 \times 16$ & $\begin{array}{c}2.7542 e+0 \\
1\end{array}$ & 3.9083 & $\begin{array}{c}2.0053 e+0 \\
1\end{array}$ & $\begin{array}{c}4.338 \\
8\end{array}$ & $\begin{array}{c}2.0135 e+0 \\
1\end{array}$ & $\begin{array}{c}4.447 \\
0\end{array}$ & $2.0022 e+01$ & 4.4515 \\
\hline $32 \times 32$ & $\begin{array}{c}4.4083 e+0 \\
1\end{array}$ & - & $1.9063 e-01$ & $\begin{array}{c}6.716 \\
9\end{array}$ & $2.1971 e-02$ & $\begin{array}{c}9.839 \\
9\end{array}$ & $1.8656 e-02$ & 10.068 \\
\hline $64 \times 64$ & $\begin{array}{c}1.9575 e+0 \\
1\end{array}$ & 1.1712 & $2.1823 e-02$ & $\begin{array}{c}3.126 \\
9\end{array}$ & $3.5110 e-04$ & $\begin{array}{c}5.967 \\
5\end{array}$ & $3.7910 e-05$ & 8.9428 \\
\hline
\end{tabular}

TABLE 10: Convergence rate of bending moment with quasi-equidistant nodes $d_{1}\left(d_{2}=7\right)$.

\begin{tabular}{|c|c|c|c|c|c|c|c|c|}
\hline$n$ & \multicolumn{2}{|c|}{$d_{1}=2$} & \multicolumn{2}{|c|}{$d_{1}=3$} & \multicolumn{2}{|c|}{$d_{1}=4$} & \multicolumn{2}{|c|}{$d_{1}=5$} \\
\hline $8 \times 8$ & $\begin{array}{c}7.5367 e+0 \\
0\end{array}$ & & $\begin{array}{c}7.9725 e+0 \\
0\end{array}$ & & $\begin{array}{c}8.6829 e+0 \\
0\end{array}$ & & $8.6637 e+00$ & \\
\hline $16 \times 16$ & $5.5240 e-01$ & 3.7701 & $4.1382 e-01$ & $\begin{array}{c}4.267 \\
9\end{array}$ & $4.1551 e-01$ & $\begin{array}{c}4.385 \\
2\end{array}$ & $4.1284 e-01$ & 4.3913 \\
\hline $32 \times 32$ & $7.3745 e-01$ & - & $3.1740 e-03$ & $\begin{array}{c}7.026 \\
6\end{array}$ & $3.7026 e-04$ & $\begin{array}{c}10.13 \\
2\end{array}$ & $3.1421 e-04$ & 10.360 \\
\hline $64 \times 64$ & $3.1548 e-01$ & 1.2250 & $1.7330 e-03$ & $\begin{array}{l}0.873 \\
0\end{array}$ & $2.2647 e-03$ & - & $5.4457 e-03$ & - \\
\hline
\end{tabular}

TABLE 11: Convergence rate of deflection with quasi-equidistant nodes $d_{2}\left(d_{1}=7\right)$.

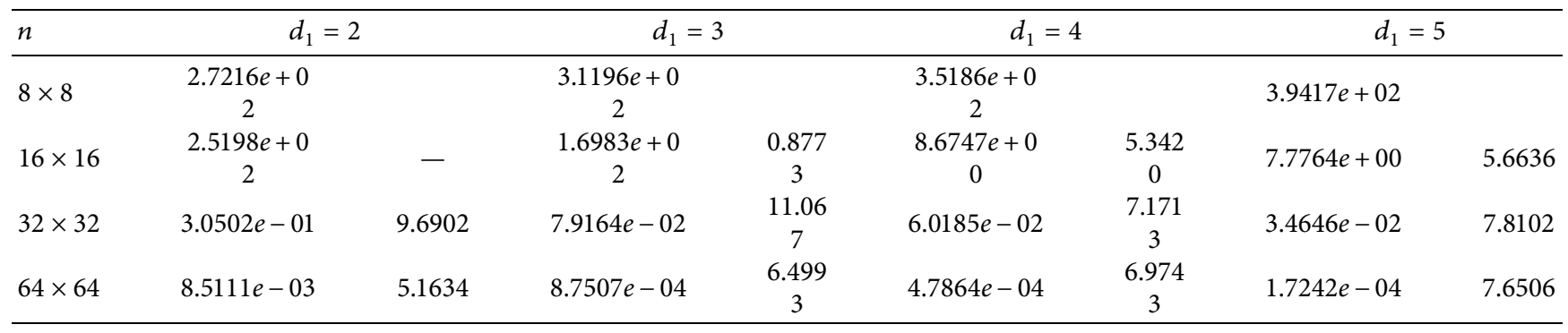


TABLE 12: Convergence rate of bending moment with quasi-equidistant nodes $d_{2}\left(d_{1}=7\right)$.

\begin{tabular}{|c|c|c|c|c|c|c|c|c|}
\hline$n$ & \multicolumn{2}{|c|}{$d_{1}=2$} & \multicolumn{2}{|c|}{$d_{1}=3$} & \multicolumn{2}{|c|}{$d_{1}=4$} & \multicolumn{2}{|c|}{$d_{1}=5$} \\
\hline $8 \times 8$ & $\begin{array}{c}5.4001 e+0 \\
0\end{array}$ & & $\begin{array}{c}6.1897 e+0 \\
0\end{array}$ & & $\begin{array}{c}6.9811 e+0 \\
0\end{array}$ & & $7.8208 e+00$ & \\
\hline $16 \times 16$ & $\begin{array}{c}5.1958 e+0 \\
0\end{array}$ & - & $\begin{array}{c}3.5019 e+0 \\
0\end{array}$ & $\begin{array}{c}0.821 \\
7\end{array}$ & $1.7887 e-01$ & $\begin{array}{c}5.286 \\
5\end{array}$ & $1.6035 e-01$ & 5.6080 \\
\hline $32 \times 32$ & $5.1369 e-03$ & 9.9822 & $1.3332 e-03$ & $\begin{array}{c}11.35 \\
9\end{array}$ & $1.0136 e-03$ & $\begin{array}{c}7.463 \\
3\end{array}$ & $5.8348 e-04$ & 8.1023 \\
\hline $64 \times 64$ & $1.7818 e-02$ & - & $4.4772 e-02$ & - & $3.3747 e-02$ & - & $2.6845 e-02$ & - \\
\hline
\end{tabular}

$$
u(x, t)=\left(1+\frac{x^{5}}{120}\right) \sin t .
$$

In this example, we test the linear barycentric rational for the equidistant nodes. Table 1 shows the convergence rate is $O\left(h^{d_{1}-1}\right)$ with $d_{2}=7$ firstly given for the space area for $t=\pi$. In Table 2, for the space area partition $d_{1}=7$ firstly given, the convergence rate of times is $O\left(\tau^{d_{2}}\right)$ which agrees with our theorem analysis.

In Figures 1 and 2, the errors of LBRCM by equidistant nodes and quasi-equidistant nodes with $m=n=12$ and $d_{1}=d_{2}=9$ are presented. From the figure, we know that the accuracy of equidistant node is higher than quasi-equidistant node.

In Figures 3 and 4, the errors of equidistant nodes and quasi-equidistant nodes with $m=n=12$ are presented. From the figure, we know that the accuracy of equidistant node is higher than the quasi-equidistant node.

In Tables 3 and 4, we test the linear barycentric rational for the quasi-equidistant nodes; Table 3 shows the convergence rate is $O\left(h^{d_{1}-1}\right)$ with $d_{2}=7$ firstly given for the space area for $t=\pi$. In Table 4 , for the space area partition $d_{1}=7$ firstly given, the convergence rate of times is $O\left(\tau^{d_{2}}\right)$.

Example 2. Consider the beam force vibration equation:

$$
\mathrm{EI} \frac{\partial^{4} w}{\partial x^{4}}+\rho \frac{\partial^{2} w}{\partial t^{2}}=Q \sin \frac{\pi x}{l} \sin (p t), \quad 0 \leq x \leq l ; t \geq 0
$$

with the boundary conditions as follows:

$$
\begin{aligned}
u(x, 0) & =0, \\
u_{t}(x, 0) & =0, \quad 0<x<l, \\
u(0, t) & =0, \\
u_{x x}(0, t) & =0, \\
u(l, t) & =0, \\
u_{x x}(l, t) & =0, \quad t \geq 0 .
\end{aligned}
$$

Its analysis solutions is

$$
\begin{aligned}
w(x, t) & =\frac{Q}{\rho} \sin \left(\frac{\pi x}{l}\right) \frac{\sin (p t)-(p / w) \sin (w t)}{w^{2}-p^{2}}, \\
w & =\pi^{2} \sqrt{\frac{\mathrm{EI}}{\rho^{4}}}, \\
M(x, t) & =\mathrm{EI} \frac{\partial^{2} w}{\partial x^{2}}=\frac{\mathrm{QEI} \pi^{2}}{\rho l^{2}} \sin \left(\frac{\pi x}{l}\right) \frac{\sin (p t)-(p / w) \sin (w t)}{w^{2}-p^{2}}, \\
w & =\pi^{2} \sqrt{\frac{\mathrm{EI}}{\rho^{4}}},
\end{aligned}
$$

where $\mathrm{EI}=4.7726 \times 10^{7}, \rho=420, Q=10^{7}, l=10$, and $p=$ $(2 \pi / 0.28335)$.

In Figures 5 and 6 , the errors of deflection with quasiequidistant nodes $m=n=28, d_{1}=d_{2}=25$, and $m=n=28$ are presented. From the figure, we know that the accuracy of quasi-equidistant node with $m=n=28$ and $d_{1}=d_{2}=25$ is higher than $m=n=28$.

In this example, we test the linear barycentric rational for deflection and bending moment with the equidistant nodes; Table 5 shows the convergence rate is $O\left(h^{d_{2}}\right)$ with $d_{1}=7$ firstly given for the space area for $t=1$. In Table 6 , for the space area partition $d_{1}=7$ firstly given, the convergence rate of times is $O\left(\tau^{d_{2}}\right)$ which agrees with our theorem analysis.

In this example, we test the linear barycentric rational for deflection and bending moment with the equidistant nodes; Table 7 shows the convergence rate is $O\left(h^{d_{2}-1}\right)$ with $d_{1}=7$ firstly given for the space area for $t=1$. In Table 8 , for the space area partition $d_{1}=7$ firstly given, the convergence rate of times is $O\left(\tau^{d_{2}}\right)$ which agree with our theorem analysis.

In Tables 9 and 10, we test the linear barycentric rational for deflection and bending moments with the quasi-equidistant nodes; Table 9 shows the convergence rate is $O\left(h^{d_{1}}\right)$ with $d_{2}=7$ firstly given for the space area for $t=1$. In Table 10 , for the space area partition $d_{2}=7$ firstly given, the 
convergence rate of times is $O\left(\tau^{d_{1}}\right)$ which agrees with our theorem analysis.

In Tables 11 and 12, we test the linear barycentric rational collocation methods for deflection and bending moment with the quasi-equidistant nodes; Table 11 shows the convergence rate is $O\left(h^{d_{1}}\right)$ with $d_{2}=7$ firstly given for the space area for $t=1$. In Table 12, for the space area partition $d_{2}=7$ firstly given, the convergence rate of times is $O\left(\tau^{d_{1}}\right)$ which agrees with our theorem analysis.

\section{Conclusion}

In this paper, linear barycentric rational collocation methods have been presented to solve the beam force vibration equation. With the help of matrix equation of discrete beam force vibration equation, the time and space variable can be solved at the same time. As the coefficient matrix is full for the collocation methods, there are certain properties such as circularity and symmetry that can be studied in the near future. The $2+1$ dimensional beam force vibration equation can also be solved easily by barycentric rational collocation methods.

\section{Data Availability}

The data that support the findings of this study are available from the corresponding author upon reasonable request.

\section{Conflicts of Interest}

The authors declare that they have no conflicts of interest.

\section{Acknowledgments}

This manuscript was written by Jin Li and some corrections on Grammar were given by Yu Sang. The work of Jin Li was supported by the Natural Science Foundation of Shandong Province (Grant no. ZR2016JL006), the Natural Science Foundation of Hebei Province (Grant no. A2019209533), and the National Natural Science Foundation of China (Grant no. 11771398).

\section{References}

[1] H. M. Numanoglu, B. Akgoz, and O. Civalek, "On dynamic analysis of nanorods," International Journal of Engineering Science, vol. 130, pp. 33-50, 2018.

[2] O. Civalek, S. Dastjerdi, S. D. Akbas, and B. Akgoz, "Vibration analysis of carbon nanotube-reinforced composite microbeams," Mathematical Methods in the Applied Sciences, 2021.

[3] Y. Chen, X. Zhao, and Y. Huang, "Mortar element method for the time dependent coupling of Stokes and Darcy flows," Journal of Scientific Computing, vol. 80, no. 2, pp. 1310-1329, 2019.

[4] X. Lin, Y. Chen, Y. Huang, and Y. Q. Huang, "A posteriori error estimates of hp spectral element methods for optimal control problems with L2-norm state constraint," Numerical Algorithms, vol. 83, no. 3, pp. 1145-1169, 2020.

[5] J. Shen, T. Tang, and L. Wang, Spectral Methods Algorithms, Analysis and Applications, Springer-Verlag, New York, NY, USA, 2011.
[6] J.-P. Berrut and L. N. Trefethen, "Barycentric Lagrange interpolation," SIAM Review, vol. 46, no. 3, pp. 501-517, 2004.

[7] P. Berrut, S. A. Hosseini, and G. Klein, "The linear barycentric rational quadrature method for Volterra integral equations," SIAM Journal on Scientific Computing, vol. 36, no. 1, pp. 105-123, 2014.

[8] J.-P. Berrut and G. Klein, "Recent advances in linear barycentric rational interpolation," Journal of Computational and Applied Mathematics, vol. 259, pp. 95-107, 2014.

[9] A. Abdi, J. P. Berrut, and S. A. Hosseini, "The linear barycentric rational method for a class of delay volterra integrodifferential equations," Journal of Scientific Computing, vol. 75, no. 3, pp. 1757-1775, 2018.

[10] J.-P. Berrut, M. S. Floater, and G. Klein, "Convergence rates of derivatives of a family of barycentric rational interpolants," Applied Numerical Mathematics, vol. 61, no. 9, pp. 989-1000, 2011.

[11] E. Cirillo and K. Hormann, "On the Lebesgue constant of barycentric rational Hermite interpolants at equidistant nodes," Journal of Computational and Applied Mathematics, vol. 349, pp. 292-301, 2019.

[12] M. S. Floater and K. Hormann, "Barycentric rational interpolation with no poles and high rates of approximation," Numerische Mathematik, vol. 107, no. 2, pp. 315-331, 2007.

[13] G. Klein and J.-P. Berrut, "Linear rational finite differences from derivatives of barycentric rational interpolants," SIAM Journal on Numerical Analysis, vol. 50, no. 2, pp. 643-656, 2012.

[14] G. Klein and J.-P. Berrut, "Linear barycentric rational quadrature," BIT Numerical Mathematics, vol. 52, no. 2, pp. 407-424, 2012.

[15] J. Li and Y. L. Cheng, "Linear barycentric rational collocation method for solving integro-differential equation," Computational and Applied Mathematics, vol. 39, p. 92, 2020.

[16] J. Li and Y. L. Cheng, "Linear barycentric rational collocation method for solving heat conduction equation," Numerical Methods for Partial Differential Equations, vol. 37, pp. 533$545,2021$.

[17] J. Li and Y. L. Cheng, "Barycentric rational method for solving biharmonic equation by depression of order," Numerical Methods for Partial Differential Equations, vol. 12, 2021.

[18] S. Li and Z. Wang, High Precision Meshless Barycentric Interpolation Collocation Method-Algorithmic Program and Engineering Application, Science Publishing, Beijing, China, 2012.

[19] Z. Wang and S. Li, Barycentric Interpolation Collocation Method for Nonlinear Problems, National Defense Industry Press, Beijing, China, 2015.

[20] Z. Wang, L. Zhang, Z. Xu, and J. Li, "Mixed barycentric interpolation collocation method of displacement-pressure for incompressible plane elastic problems," Chinese Journal of Applied Mechanics, vol. 35, no. 2, pp. 195-201, 2018.

[21] Z. Wang, L. Zhang, Z. Xu, and J. Li, "Barycentric interpolation collocation method based on mixed displacement-stress formulation for solving plane elastic problems," Chinese Journal of Applied Mechanics, vol. 35, no. 2, pp. 304-309, 2018. 